\title{
A JOINT CALIBRATION METHOD OF 3D IMAGES ACQUIRED BY COMMON OPTICAL PATH PAYLOAD
}

\author{
G.E. Teng ,M. Zhou*, J. Hu, H.H. Wu, W. Li , H.J. Zhang , J.Y. Chen , J.H. Wang
}

Key Laboratory of Quantitative Remote Sensing Information Technology, Academy of Opto-Electronics, Chinese Academy of Sciences, Beijing 100094, China - (tenggeer, zhoumei, jhu, hhwu, liwei, hjzhang, jychen)@ aoe.ac.cn, jinhu.wang@tudelft.nl

KEY WORDS: Calibration, Common Optical Path, Least Squares Principle, Misalignment Error, Ranging Error

\begin{abstract}
:
The common optical path payload is a new type of imaging payload that can acquire LiDAR data and CCD images simultaneously. This new payload integrates the linear LiDAR and linear CCD images according to the common optical system, and achieves the registration of LiDAR point cloud and CCD images by the alignment of common aperture optical axis and time synchronization control in the front of hardware. Based on the fixed matching relationship between CCD probes and LiDAR probes offered by optical path of common, this paper proposes a joint calibration method, which reduces the ranging error and misalignment error. And the results verify that this method can effectively improve the quality of the three-dimensional images. The standard deviations of validation area and roof are improved from $0.54 \mathrm{~m}$ to $0.14 \mathrm{~m}$ and $1.83 \mathrm{~m}$ to $0.26 \mathrm{~m}$ respectively.
\end{abstract}

\section{INTRODUCTION}

Airborne LiDAR can quickly obtain the three-dimensional coordinates of the objects, but it is difficult to get the textures. CCD camera can acquire the texture information of the object, with high resolution and rich details of target characteristics, which give a complement to the discrete three-dimensional point clouds (Zhang, 2012). Therefore, the combination of CCD images information and point cloud data to generate 3D images can combine the advantages of these two data sources.

The common optical path payload is a new type of imaging payload that can acquire LiDAR data and CCD images simultaneously. This new payload integrates the linear LiDAR and linear CCD images according to the common optical system, and achieves the registration of LiDAR point cloud and CCD images by the alignment of common aperture optical axis and time synchronization control in the front of hardware. Based on the above matching relation, the CCD images and the LiDAR point cloud can be fused rapidly to generate $3 \mathrm{D}$ images, the Figure 1 shows the schematic diagram of common optical path payload (Zhang, 2015).

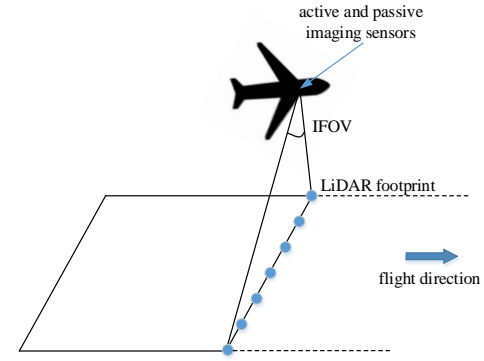

Figure 1. The schematic diagram of common optical path payload

Since laser scanners had been introduced into the field of mapping in 2000. The precision; accuracy of point clouds and the instruments calibration had become a hot topic (Medie, 2017; Xu, 2015; Xu, 2016; Li, 2011; Xia, 2016; Zhang, 2011; Guo, 2012).
Zhan et al. (2016) proposed the radiation calibration model of LiDAR. DocJana et al. (2010) discussed the influences of reflectivity, colour and other factors on the distance measurement by different colours targets. Briese et al. (2012) studied influences of the distances, colours, incident angles and materials. The above methods obtain the ranging error values of serval colour targets with different reflectivity, but no further study is made on the field of ranging error correction. Zheng et al. (2015) analysed the distance measurement factors of laser scanner. Liu el al. (2009) provided the reference models for a variety of laser scanners. Wang et al. (2010) made the distance parameters calibration for RA-360 laser scanner. Yang et al. led additive, multiply and grayscale correction into the domestic laser scanner ranging. And compared the distance measure accuracy between the grayscale priority model and the additive and multiply constant priority model.

The airborne LiDAR system calibration mainly includes IMU misalignment calibration $(\Delta \omega ; \Delta \varphi ; \Delta \kappa)$, GPS misalignment calibration $(\Delta X ; \Delta Y ; \Delta Z)$, laser scanner's ranging and angle measurement calibration $(\Delta \rho ; S \rho ; \Delta \beta ; S \beta)$. The calibration of these parameters for airborne LiDAR is generally eliminated by ground static calibration and flight dynamics calibration.

Ground static calibration are always operated by the manufacturers, mainly for individual equipment. Vaughn et al. (1996) and Favey (2001) verified the measured distance, GPS phase centre and laser scanner's geometric. Meanwhile Academy of Opto-electronics of CAS, Chinese Academy of Surveying \& Mapping, Information Engineering University also made a study of relevant research on airborne LiDAR ground static calibration with the development of domestic airborne LiDAR (Wang, 2011; Ma, 2011).

IMU misalignment error is the largest error source in the airborne LiDAR. It is hard to measure directly, and is easily to be affected by equipment assembled and environment. So IMU misalignment calibration is essential in every flight mission and eliminated by flight dynamic calibration. Some researchers treat

\footnotetext{
* Correspondence: zhoumei@aoe.ac.cn; Tel.: +86-010-8217-8634
} 
the ranging errors and GPS misalignment errors as unknown parameters for flight dynamic calibration.

The LiDAR flight dynamic calibration are mainly based on point clouds. To be specific, it mainly uses some special outlines of targets, the targets position deviations (obtained by different stripes) based on point cloud coordinates calculate equations are solved, then calculates the parameters according to the same coordinates or other constraints. Bang et al. (2008) believed that the terrestrial laser scanner has the advantages in high density and high precision compared to the airborne laser scanner, which could be used as a reference dataset for misalignment calibration. Filin (2003) used natural surfaces or artificial targets to calculate the calibration parameters. Skaloud et al. (2006) used coplanar features to solve the misalignment angle error and ranging error, according to error functions which involved the coplanar parameters and system error parameters. Hebel et al. (2012) used the region growth and RANSAC to extract planes to solve error parameters. Habib et al. (2007a; 2007b) extracted planes from the images and added the point cloud equations to adjustment equations. This method not only solved the mislignment parameters, but also registrated the airborne point clouds to the images. The methods mentioned above were only for laser scanners, there isn't common optical path equipment was studied. Based on the fixed matching relationship between CCD probes and LiDAR probes offered by optical path of common, this paper proposes a joint calibration method based on ground static and flight dynamics for common aperture optical load under the premise of parallel optical axis, which reduces the ranging error and misalignment error. According to the data in the building area, the results verify that this method can effectively improve the quality of 3D images.

\section{IMAGING PRINCIPLE OF COMMON APERTURE OPTICAL LOAD}

Common optical path payload integrated a linear LiDAR and a linear CCD to perform push-broom imaging of the target by common optical path system. The imaging principle has been shown in Figure 2. The common optical path payload achieved the correspondence between the laser probes and the CCD pixels perpendicular to the flight direction by alignment of common aperture optical axis, meanwhile, the usage of time synchronization control ensured correspondence between the laser scanning lines and the CCD scanning lines along the flight direction.

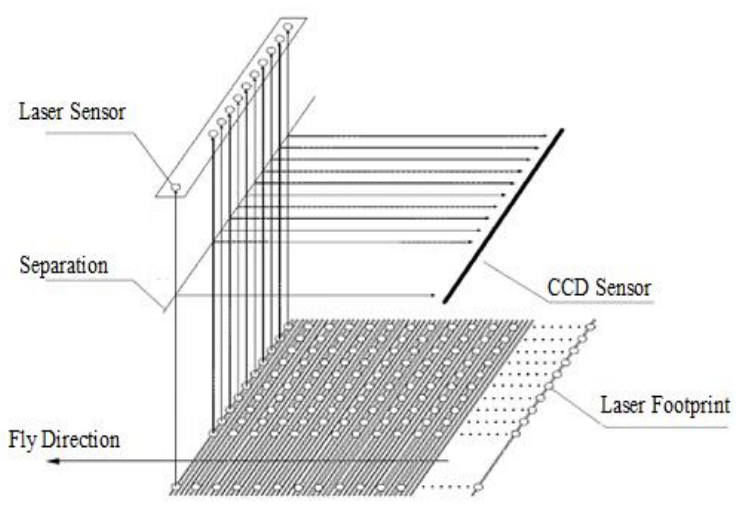

Figure 2. Common optical path payload imaging principle

\section{JOINT CALIBRATION METHOD}

Based on the characteristics of this payload, ensuring the accurate matching relation of linear LiDAR and linear CCD is the key to obtain a high-precision 3D colour point clouds, therefore optical axis parallelism calibration is done before the joint calibration Firstly, the ground static calibration solves the additive and multiply constant of each laser receiving unit based on the sixsegment analytical method. Then, according to the high-precision matching relationship between linear laser radar and linear array $\mathrm{CCD}$, the ground truth value coordinate points are used for dynamic calibration based on the least squares principle, and the misalignment error are solved. Finally, the calibration results are verified by the real three-dimensional feature information in the survey area. The processing flow is shown in Figure 3.)

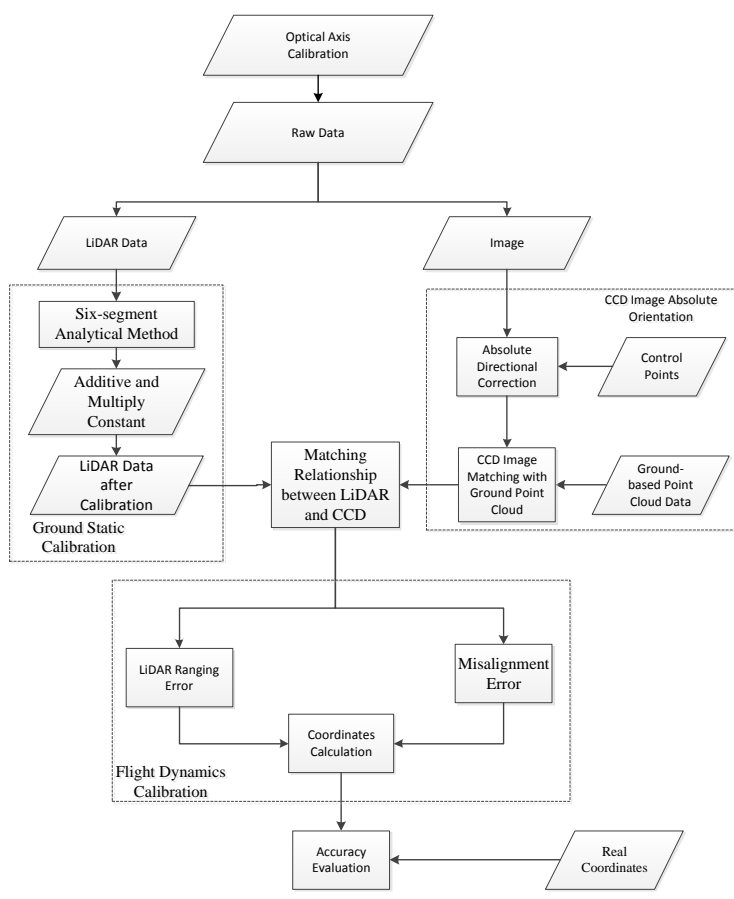

Figure 3. Processing Flow

\subsection{Parallelism Calibration of Optical Axis}

Optical axis parallelism calibration is mainly based on indoor optical axis alignment debugging method of long-focus optical focal plane imaging. The main principle is that, for different parallel lights, with different angles between the light and optical axis, when the parallel light gets into the collimator, it will be focused on the different positions at focal plane. On the contrary, if the imaging facula of the optical light representing different sensor receives coincidence in the collimator focal plane, then the central axis of the sensors field of view are considered to be parallel. Then repeated the calibration to the other detectors (Zhang, 2015). The calibration system is shown in Figure 4.

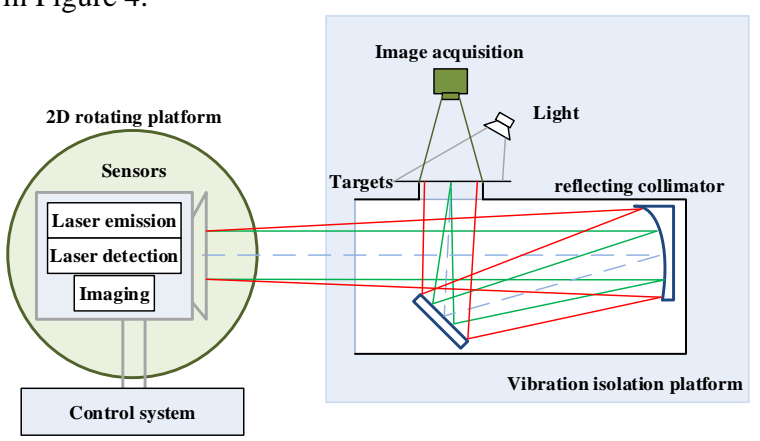

Figure 4. Schematic diagram of calibration system 


\subsection{Special Target}

In order to capture the laser footprint on the ground for sixsegment analytical method successfully, we designed a detachable laser footprint target. The target plates are installed into the frame, which is made of steel, target plates are made of wood. This special target can solve the problem of huge laser footprint is hard to find, which caused by the long distance. The detachable targets are shown in the Figure 5.

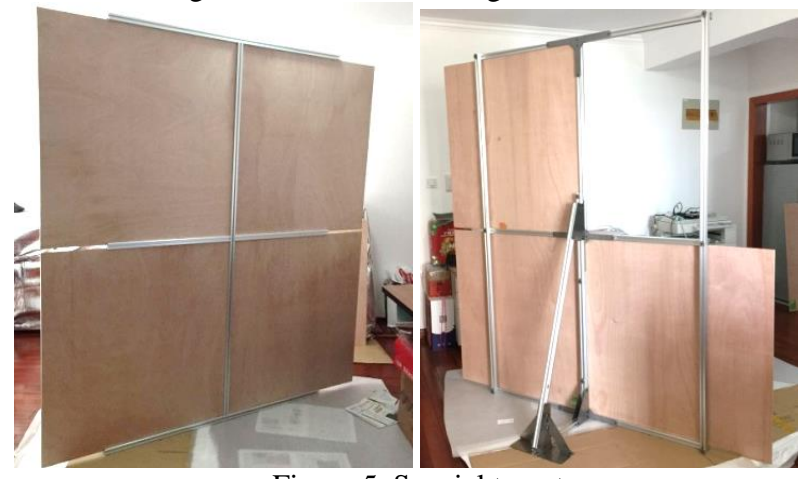

Figure 5. Special target

\subsection{Ground Static Calibration}

There are many factors that affect the accuracy of laser ranging, such as time delay of the equipment's internal circuit, atmospheric refraction, different surface reflectors and so on. The time delay mainly presented as additive and multiply error of distance measurement, and the ranging error caused by atmospheric refraction is only in millimetre level, which is much smaller than the ranging accuracy. Therefore, laser ranging calibration consists of the additive and multiply ranging error calculation.

The ground static calibration adopts the six-segment analytical method, and seven markers are arranged on a straight line. Lidar devices are installed on these seven points for scanning, and 21 distance values are obtained. Finally, the normal equation is established by using the ranging value of each line segment and the total station distance measurement value, finally, the additive and multiply constant are calculated, just as the Figure 6. shows.

$$
L_{Q}=L_{L}+K_{0}+K_{1} L_{L}
$$

where $L_{Q}=$ the distance value of the total station

$L_{L}=$ the distance value from the laser emission center to the mark point

$$
\begin{aligned}
& K_{0}=\text { distance additive constant value } \\
& K_{1}=\text { distance additive multiply value }
\end{aligned}
$$

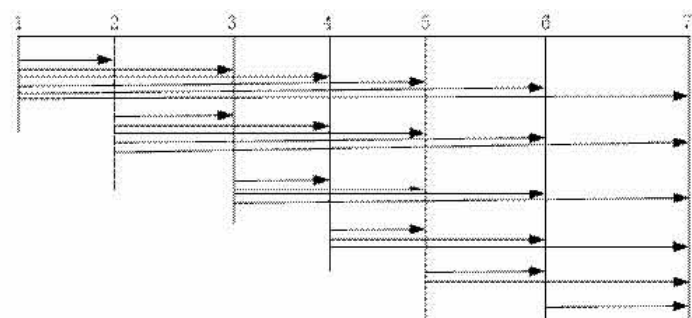

Figure 6. Six-segment analytical method

The additive and multiply constant error are calculated based on the least square method by measuring the ranging value every unit. The initial additive and multiply value are brought into the No. I laser ranging value, the observation value is compared with the known baseline value.

$$
\left.\begin{array}{c}
D_{01}+v_{01}+K_{0}+D_{01} K_{1}=\bar{D}_{01} \\
D_{02}+v_{02}+K_{0}+D_{02} K_{1}=\bar{D}_{02} \\
D_{56}+v_{56}+K_{0}+D_{56} K_{1}=\bar{D}_{56}
\end{array}\right\}
$$

where $\quad D_{01} \sim D_{56}=$ initial distance value after additive and multiply adjust

$v_{01} \sim v_{56}=$ distance value adjustment value

$\bar{D}_{01} \sim \bar{D}_{56}=$ distance value after adjustment

In error equations, $l_{01} \sim l_{56}$ is the differences between baseline values and observation values, for example, $l_{01}=\bar{D}_{01}-D_{01}$

$$
\left.\begin{array}{l}
v_{01}=-K_{0}-D_{01} K_{1}+l_{01} \\
v_{02}=-K_{0}-D_{02} K_{1}+l_{02} \\
v_{56}=-K_{0}-D_{56} K_{1}+l_{56}
\end{array}\right\}
$$

where $l_{01} \sim l_{56}=$ the differences between baseline and observation

$$
\left.\begin{array}{c}
21 K_{0}+[D] K_{1}-[l]=0 \\
{[D] K_{0}+[D D] K_{1}-[D l]=0}
\end{array}\right\}
$$

where $\quad K_{0}=$ distance additive constant value

$K_{1}=$ distance additive multiply value

Thereby, the additive constant $K_{0}$ and the multiply constant $K_{1}$ can be solved.

\subsection{Consistency Verification}

(1) The payload worked toward the wall, and output the ranging values of every detector.

(2) The consistency verification of every detector was done after additive and multiply adjustment. Figure 7. and Figure 8. show the point cloud results before and after adjustment. The root mean squared error was improved from $\pm 2.173 \mathrm{~m}$ to $\pm 1.088 \mathrm{~m}$.

Figure 7. Point cloud result before adjustment

Figure 8. Point cloud result after adjustment

From the above results, it can be seen that the root mean squared error of the wall after calibration is lower than the ones before calibration, which demonstrated the effectiveness of the ground static calibration. The additive and multiply constant values of distance measurement proved to be effective to improve the accuracy of point cloud processing.

\subsection{Flight Dynamics Calibration}

In order to verify the common optical path payload's performance, the flight trial was carried out in BaiMajing Town, Danzhou City, Hainan Province. The flight area coordinates are 
listed in Table 1., the place used for flight dynamics calibration is also in the area, as shown in the Figure 9.

\begin{tabular}{|c|c|}
\hline Longitude & Latitude \\
\hline $109^{\circ} 07^{\prime} 24^{\prime}, \mathrm{E}$ & $19^{\circ} 38^{\prime} 37^{\prime} \mathrm{N}$ \\
$109^{\circ} 08^{\prime} 08^{\prime}, \mathrm{E}$ & $19^{\circ} 37^{\prime} 38^{\prime \prime} \mathrm{N}$ \\
$109^{\circ} 12^{\prime} 51^{\prime}, \mathrm{E}$ & $19^{\circ} 42^{\prime} 47^{\prime} \mathrm{N}$ \\
$109^{\circ} 133^{\prime} 35^{\prime} \mathrm{E}$ & $19^{\circ} 41^{\prime} 48^{\prime} \mathrm{N}$ \\
\hline
\end{tabular}

Table 1. Flight area coordinates
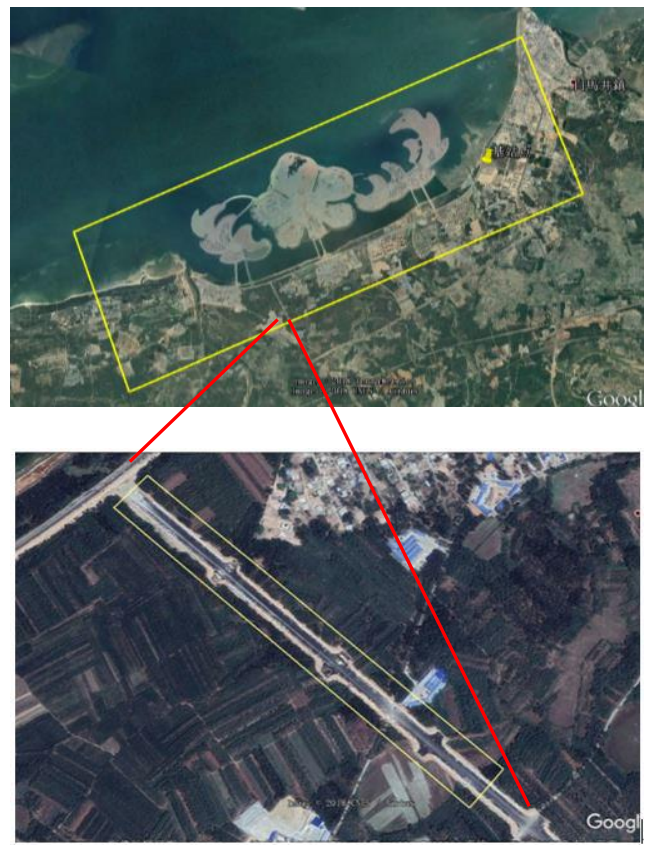

Figure 9. Fight area and the place used for flight dynamics calibration

The joint calibration of CCD detector and LiDAR refers to the registration that is between the higher ground resolution of the linear array $\mathrm{CCD}$ and the $3 \mathrm{D}$ point cloud of the real on the ground. The specific process is as follows:

1. Improving the absolute accuracy of $\mathrm{CCD}$ image pixels by image control points.

2. Calculating the ground-based feature points' accurate position in the CCD image, just as shown in figure 3 , therefore calculating the linear $\mathrm{CCD}$ probe corresponding to the ground-based feature point;

3. Through the CCD probes and LiDAR probes of the common optical path (5:1), the corresponding LiDAR probe is determined; 4. By the ground-based LiDAR feature points, LiDAR ranging error and misalignment error can be solved by the least squares method.

The 5 feature points of CCD images are listed in Figure 10., the positions of CCD probes in the ground LiDAR point cloud coule be determined by 5 feature points, and then the corresponding LiDAR probes are determined.

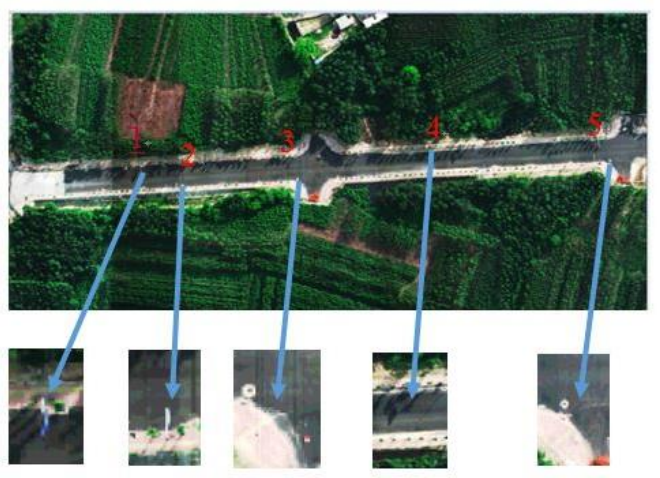

Figure 10. Five feature points of CCD images

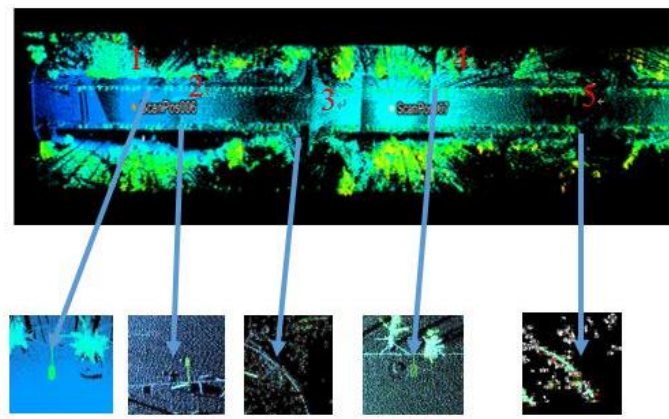

Figure 11. Ground-based LiDAR Feature Points

\begin{tabular}{|c|c|c|}
\hline $\begin{array}{c}\text { Feature } \\
\text { Point }\end{array}$ & $\begin{array}{c}\text { CCD } \\
\text { Probes }\end{array}$ & $\begin{array}{c}\text { LiDAR } \\
\text { Probes }\end{array}$ \\
\hline 1 & 486 & 77 \\
2 & 594 & 99 \\
3 & 974 & 175 \\
4 & 1449 & 270 \\
5 & 1889 & 358 \\
\hline
\end{tabular}

Table 12. 5 Feature Points' CCD and LiDAR Probes

The airborne LiDAR calculates the high-precision laser footprint coordinates according to the distance $D$, splitting angle $\theta$, the attitude and position of the aircraft.

The calculation needs to consider the optical device centre, the laser emission position, the GPS antenna position, the positional offset and rotation between the optical path imaging load and the GNSS. The strict geometric calibration model is as follows:

$$
\begin{gathered}
{\left[\begin{array}{l}
x_{\text {spot }} \\
y_{\text {spot }} \\
z_{\text {spot }}
\end{array}\right]=R_{W} R_{G} R_{N}\left[R_{M} D\left[\begin{array}{c}
0 \\
\sin \theta \\
-\cos \theta
\end{array}\right]+\left[\begin{array}{l}
\Delta X_{I M U} \\
\Delta Y_{I M U} \\
\Delta Z_{I M U}
\end{array}\right]-\right.} \\
\left.\left[\begin{array}{l}
\Delta X_{G P S} \\
\Delta Y_{G P S} \\
\Delta Z_{G P S}
\end{array}\right]\right]+\left[\begin{array}{l}
\Delta x_{\text {spot }} \\
\Delta x_{\text {spot }} \\
\Delta x_{\text {spot }}
\end{array}\right] \\
\text { where } \quad\left[\begin{array}{l}
x_{\text {spot }} \\
y_{\text {spot }} \\
z_{\text {spot }}
\end{array}\right]=\text { laser footprint in WGS-84 coordinate system } \\
{\left[\begin{array}{l}
\Delta x_{\text {spot }} \\
\Delta y_{\text {spot }} \\
\Delta z_{\text {spot }}
\end{array}\right]=\text { GPS centre in WGS-84 coordinate system }} \\
R_{W} \text { R } R_{G}=\text { transition matrix relevant to current location } \\
R_{N}=\text { attitude angle rotation matrix } \\
R_{M}=\text { IMU misalignment error rotation matrix }
\end{gathered}
$$




$$
\left[\begin{array}{c}
0 \\
D \sin \theta \\
-D \cos \theta
\end{array}\right]=\text { laser beam coordinates in instantaneous }
$$

emission coordinate

$$
\begin{aligned}
& {\left[\begin{array}{l}
\Delta X_{I M U} \\
\Delta Y_{I M U} \\
\Delta Z_{I M U}
\end{array}\right]=\text { GPS misalignment offset }} \\
& {\left[\begin{array}{l}
\Delta X_{G P S} \\
\Delta Y_{G P S} \\
\Delta Z_{G P S}
\end{array}\right]=\text { GPS phase centre misalignment offset }}
\end{aligned}
$$

In the formula,

$R_{M}=$

$\left[\begin{array}{ccc}1 & 0 & 0 \\ 0 & \cos \alpha & -\sin \alpha \\ 0 & \sin \alpha & \cos \alpha\end{array}\right]\left[\begin{array}{ccc}\cos \beta & 0 & \sin \beta \\ 0 & 1 & 0 \\ -\sin \beta & 0 & \cos \beta\end{array}\right]\left[\begin{array}{ccc}\cos \gamma & -\sin \gamma & 0 \\ \sin \gamma & \cos \gamma & 0 \\ 0 & 0 & 1\end{array}\right]$ (5) Based on the principle of minimizing the residual of the laseremitting point to the ground laser point, the error equation can be listed as

$$
\begin{gathered}
\Delta D=d D+\frac{d D}{d \alpha} d \alpha+\frac{d D}{d \beta} d \beta+\frac{d D}{d \gamma} d \gamma \\
V=B X-L
\end{gathered}
$$

In the formula, $\mathrm{B}=\left[\begin{array}{llll}1 & \frac{d D}{d \alpha} & \frac{d D}{d \beta} & \frac{d D}{d \gamma}\end{array}\right] ; L=\left(D-D_{0}\right)^{T} ; X=$ $\left[\begin{array}{llll}d D & d \alpha & d \beta & d \gamma\end{array}\right]$.

where $\quad D=$ laser ranging value

$D_{0}=$ approximate value of the previous calculation

The ranging error and misalignment error are solved by the least squares method.

\section{RESULTS}

The standard deviation of the elevation residual is used to evaluate the LiDAR elevation accuracy, and the ground-based measured point cloud elevation value is used as the true value to evaluate the elevation accuracy of the laser geometric model solution. All the laser points in the validation area and roof are calculated, as shown in the figure below, and standard deviations of the elevation residual before and after calibration are compared.

$$
\sigma=\sqrt{\frac{\sum_{i=1}^{N}\left(h_{i}-\bar{h}\right)^{2}}{N}}
$$

where $\quad h_{i}=$ laser footprint height value

$\bar{h}=$ validation area and roof true height value

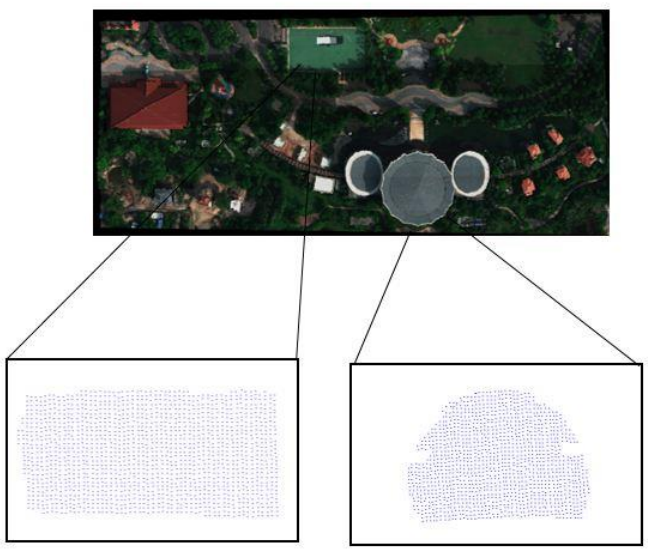

Figure 4. Validation area and roof
The effect of the validation area and roof before and after the calibration is shown in the figure below.

\begin{tabular}{|c|c|c|c|}
\hline Class & $\begin{array}{c}\text { Average } \\
\text { Elevation } / \mathrm{m}\end{array}$ & $\begin{array}{c}\text { Before } \\
\text { Calibration } / \mathrm{m}\end{array}$ & $\begin{array}{c}\text { After } \\
\text { Calibration } / \mathrm{m}\end{array}$ \\
\hline $\begin{array}{c}\text { Validation } \\
\text { Area } \\
\text { Roof }\end{array}$ & 13.83 & 0.54 & 0.14 \\
\hline
\end{tabular}

Table 2 Joint Calibration Results

Figure 5 Effect of the validation area before and after the calibration

Figure 6 Effect of the roof before and after the calibration

\section{CONCLUSIONS}

Based on the fixed relationship between point cloud and images obtained by the common optical path payload, this paper proposes a joint calibration method combining the ground static calibration method and flight dynamics calibration on the basis of parallelism calibration of optical axis, which reduces the laser radar ranging error and misalignment error in the common aperture optical payload. And the results verify that this method can effectively improve the quality of the three-dimensional images.

\section{ACKNOWLEDGMENTS}

The work is supported by the [The Science and Technology Innovation Project of the Chinese Academy of Science] under Grant [No. Y70X23A34Y].

This work was supported by National High Tech Research and development program (863 Program) of the People's Republic of China (2015AA123801).

\section{REFERENCES}

Bang, K.I., Habib, A.F., Kusevic, K., 2008. Integration of terrestrial and airborne LiDAR data for system calibration. Proceedings of The International Archives of the Photogrammetry, Remote Sensing and Spatial Information Sciences. Beijing, China, 37(part B1), 391-398.

Bang,K.I., 2010. Alternative Methodologies for LiDAR System Calibration. Calgary, University of Calgary.

Briese, C., Pfennigbauer, M., Lehner, H., 2012. Radiometric calibration of multi-wavelength airborne scanning data. ISPRS Ann. Photogramm. Remote Sens Spat Inf Sci, 1-7,335-340. 
Favey, E., 2001. Investigation and improvement of airborne laser scanning technique for monitoring surface elevation changes of glaciers. Mitteilungen Institut fur Geodasie und Photogrammetrie an der Eidgenossischen Technischen Hochschule Zurich.

Filin, S., 2003. Recovery of systematic biases in laser altimetry data using natural surfaces. Photogrammetric Engineering \& Remote Sensing, 69(11), 1235-1242.

Guo, Y.L.,Wan,J.W., Lu, M., 2012. Three dimensional orientation estimation for ladar target. Optics and Precision Engineering, 20(4), 843-850.

Habib, A.F., Bang, K., Aldelgawy, M., 2007a. Integration of photogrammetric and LiDAR data in a multi-primitive triangulation procedure. Proceddings of ASPRS 2007 Annual Conference - Identifying Geospatial Solutions. Florida.

Habib, A.F., Bang, K.I., Shin, S.W., 2007b.LiDAR system selfcalibration using planar patches from photogrammetric data. The 5th Inetrnational Symposium on Mobile Mapping Technology. Citeseer.

Hebel, M., Stilla, U., 2012. Simultaneous calibration of ALS systems and alignment of multiview LiDAR scans of urban areas. IEEE Transactions on Geoscience and Remote Sensing, 50(6), 2364-2379.

Liu, C., Zhang,Y.L., Wu, H.B.,2009. Accuracy evaluation of 3D laser range scanner based on field calibration. Geotechnical Investigation \& Surveying, 13(11),56-60.

Li, M., Song, Y.S., Yu, J.,2011.High precision laser pulse distance measuring technology. Infrared and laser Engineering, 40(8), 1469-1473.

Mazalova, J., Valentova, K., Vlckova, L., 2010. Testing of accuracy of reflectorless distance measurement of selected Leica and Topcon total stations. GeoScience Engineering, 56(1),19-26.

Ma, H, 2011. Research of error calibration methods of airborne LiDAR system. Qingdao, Shandong University of Science and Technology.

Medie, T., Holst, C., Kuhlmann, H., 2017. Towards system calibration of panoramic laser scanners from a single station. Sensors, 17(32), 11-45.

Skaloud, J., Lichti, D.,2006. Rigorous approach to bore-sight selfcalibration in airborne laser scanning. ISPRS Journal of Photogrammetry and Remote Sensing, 61(1), 47-59.

Vaughn, C.R., Button, J.L., Krabill, W.B., 1996. Georeferencing of airborne laser altimeter measurements. International Journal of Remote Sensing, 17(17), 2185-2200.

Wang, L.Z., Han, Y.M., Zhong, R.F., 2010.The range calibration of the vehicular laser scanner. Bulletin of Surveying and Mapping, 12(1), 19-20

Wang, L.C., 2011. A Study on the calibration of single LiDAR Instrument. Zhengzhou, Information Engineering University.

Xu, Z.P., Shen, H.H., Xu, Y.S.,2015.Review of the development of laser active imaging system with direct ranging. Chinese Optics. 2015, 8(1), 28-38.
Xu, Z.P., Shen, H.H., Yao, Y., 2016. Scanner less laser active imaging validating system by directly ranging. Optics and Precision Engineering, 24(2), 251-259.

Xia, W.Z., Han, S.K., Cao, J.Y., 2016. Ladar range estimation technology. Infrared and laser Engineering, 45(9), 0906005.

Yang, S.J., Zhang, K.S., Shao, Y.S., 2019. Ranging calibration model of terrestrial laser scanner based on gray level priority. Infrared and Laser Engineering, 48(01), 134-139.

Zhang, J., Zhang, L., Zheng, F.,2011.Development status of airborne 3D imaging lidar system. Chinese Optics, 4(3),213-232.

Zhang, Y., Xiong, X., Shen, X., 2012. Automatic registration of urban aerial imagery with LiDAR data. Journal of Remote Sensing, 16(3), 579-595.

Zhang, H.J., Zhou, M., Wu, H.H., Zhang, D.D., 2015. Application of high-precision matching about multisensor in fast stereo imaging. SPIE Remote Sensing, Toulouse, France, Proceedings Volume 9639, Sensors, Systems, and Next-Generation SatellitesXIX; 963928

Zheng, D.H., Chen, Y.Z., Liu, C., 2015. 3D laser scanner and its effect factor analysis of surveying error. Engineering of Surveying and Mapping, 14(2), 32-34.

Zhan, L., Alan, H.S., David, L.B., 2016. Radiometric calibration ofa dual-wavelength full-waveform terrestrial lidar. Sensors, 16(3),313. 